\title{
Cost-Estimating for Commercial Digital Printing
}

\author{
Malcolm G. Keif \\ California Polytechnic State University, San Luis Obispo, CA USA 93407
}

\begin{abstract}
The purpose of this study is to document current cost-estimating practices used in commercial digital printing. A research study was conducted to determine the use of cost-estimating in commercial digital printing companies. This study answers the questions: 1) What methods are currently being used to estimate digital printing? 2) What is the relationship between estimating and pricing digital printing? 3) To what extent, if at all, do digital printers use fullabsorption, all-inclusive hourly rates for estimating?

Three different digital printing models were identified: 1) Traditional print providers, who supplement their offset presswork with digital printing for short-run color and versioned commercial print; 2) "Low-touch" print providers, who leverage the power of the Internet to streamline business transactions with digital storefronts; 3) Marketing solutions providers, who see printing less as a discrete manufacturing process and more as a component of a complete marketing campaign. Each model approaches estimating differently.

Understanding and predicting costs can be extremely beneficial. Establishing a reliable system to estimate those costs can be somewhat challenging though. Unquestionably, cost-estimating digital printing will increase in relevance in the years ahead, as margins tighten and cost knowledge becomes increasingly more critical.
\end{abstract}

Keywords: cost-estimating, estimating, digital printing, budgeted hourly rate, BHR, MIS

\section{INTRODUCTION}

In recent years, significant attention has been given to marketing and pricing strategies for commercial digital printing. Much discussion has focused on the benefits of variable-data-printing (VDP), particularly in relation to improving direct marketing response rates. New sales models based on cost-per-response have replaced older cost-per-piece pricing strategies.

With significant profit potential for VDP campaigns, many companies focus their attention solely on marketing efforts and creating complex pricing models, with little attention given to estimating job costs prior to production. Do digital printing companies know, or even care about, their production costs on a job-by-job basis? If so, how do digital printing companies estimate individual job costs in preparation for a price quotation?

\subsection{Purpose of the study}

The purpose of this study is to document and analyze current cost-estimating practices for commercial digital printing. This research evaluates the relationship of traditional cost-estimating practices to alternate methods.

This study answers the questions: 1) What methods are currently used to estimate digital printing? 2) What is the relationship between estimating and pricing digital printing? 3) To what extent, if at all, do digital printers use fullabsorption, all-inclusive hourly rates for estimating?

Document Recognition and Retrieval XIV, edited by Xiaofan Lin, Berrin A. Yanikoglu Proc. of SPIE-IS\&T Electronic Imaging, SPIE Vol. 6500, 65000M, @ 2007 SPIE-IS\&T · 0277-786X/07/\$15 


\subsection{Relevance of cost-estimating}

Is cost-estimating relevant to digital printing? Why not simply price estimate, using simplified formulas with built-in profit to calculate price? In some cases, the customer can price the work directly by referencing a pricing matrix. For highly standardized products, price estimating makes sense. Many digital print manufacturers produce standard sizes, on standard paper, with standard inks and toners. When simple, low-complexity work is produced, pricing tables make sense, whether published or private.

When complicated, valued-added processes are involved, however, cost-estimating is helpful in managing the pricing and production functions. Variable-data, digital asset management, digital coatings, variable-finishing, or other nonstandard processes all increase the complexity and unpredictability of a job. A customized cost-estimate is helpful.

\subsection{The estimating process}

Cost-estimating involves two steps: planning production and assigning times and costs to the plan. Since cost centers have different hourly cost rates, this must be done independently for each area of production. The most commonly used estimating formula is:

$$
\text { Cost Center estimate }=(\text { production time } \times \text { BHR })+\text { Consumables }+ \text { Buyouts }
$$

Budgeted hourly rates (BHRs), or more precisely all-inclusive budgeted hourly rates, are used to apply costs to production times. The basis of all-inclusive budgeted hourly rates is the prorating of all business costs so that production rates recover both direct manufacturing costs and a portion of indirect and overhead costs. The BHR is a full-absorption cost accounting method, meaning that indirect business costs are absorbed through production rates.

The BHR for each cost center includes direct labor, equipment, supplies, and the space and utilities for the operation. It also includes a portion of the indirect costs such as indirect labor and general and administrative (G \& A) overhead. Though not a perfect science, the principle is that all costs of the business are recovered at the end of the year through the various production cost centers. All business expenses are charged to production cost centers.

Digital print producers may operate at lower capacity than commercial lithographers. This is especially true for printers specializing in on-demand products, who must have capacity available to sell at all times. Capacity fluctuations complicate the development of BHRs.

\section{METHODOLOGY}

This study used Elite and Specialized Interviewing, a technique developed by communication scholar Lewis A. Dexter as a qualitative research method of interviewing people who are highly knowledgeable on a subject. The process involves a discussion of the topic during which responses to the questions are developed. Interview questions used during the discussion were written in advance, but whenever possible, follow-up and related questions were used to discover additional information.

Thirteen interviews were conducted during the period February 1, through June 30, 2006. All interviewees were seniorlevel or estimating-level managers.

\section{RESEARCH FINDINGS}

The telephone interviews followed a structured set of questions but were tailored during the interview in the form of a discussion. Interviews averaged 30 minutes in length. The interviews focused on three areas:

- Pricing matrix and contribution to overhead

- Cost-estimating using BHRs

- Estimating specific operations 


\subsection{Pricing matrix and contribution to overhead}

The first questions asked during the interviews were:

- $\quad$ Do you use a published or private digital printing pricing matrix [price list]?

- Do you know with confidence, precisely how much each digital print job contributes to general and administrative overhead?

All participants used some form of digital printing price list with some or all customers. In some cases the lists were public but in most cases the lists were private, used for internal pricing only, and not shared with customers. Lists are predominantly used for standardized, low-complexity work.

Few printers, whether digital or conventional, can honestly answer yes to this question. To date, profit margins for digital printing have been high enough to ignore cost calculations and contributions-to-overhead. Many expressed enough profit to not be concerned with contribution on a job-by-job basis.

More than half of the respondents did not know exactly how each job contributes to General and Administrative overhead. Fifty four percent of the participating companies lacked a comprehensive costing model. Most participants knew that their work was profitable, but not how each job contributes to overhead. See Figure 1.

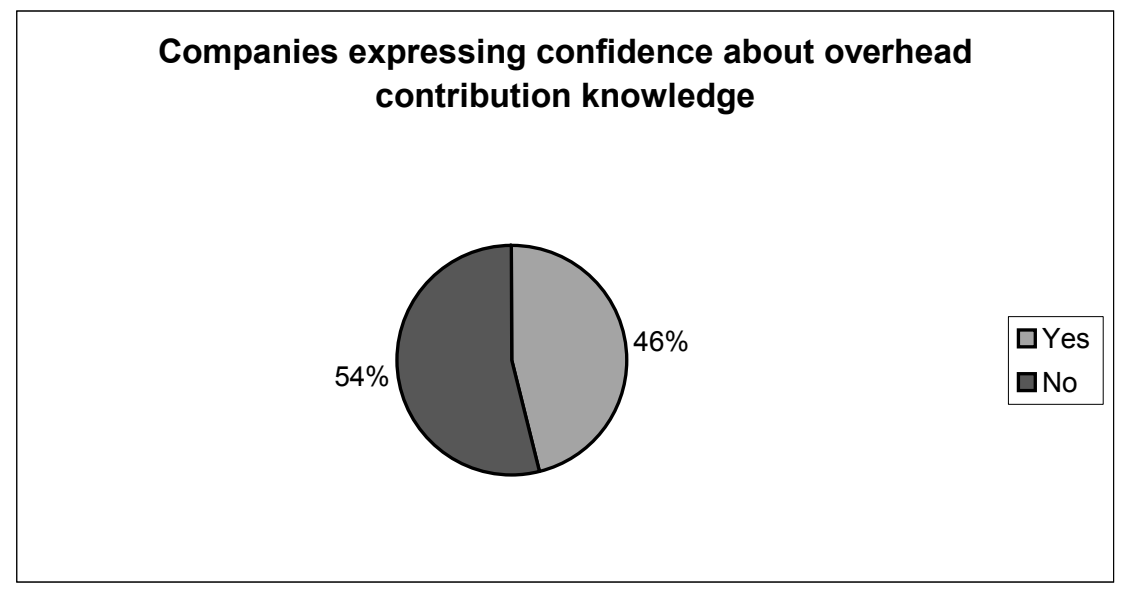

Fig. 1. Percentage of companies who knew with confidence how each digital printing job contributes to overhead..

\subsection{Cost-estimating using budgeted hourly rates (BHRs)}

The next questions were:

- Have you established an hourly cost rate or BHR for your digital press(es)?

- What percentage of your jobs involves a custom cost-estimate?

These questions focused on whether conventional cost-estimating is used for digital printing. Since several of the participants had a background in commercial printing, cost rates were largely full-absorption BHRs. When asked if hourly rates had been established for digital presses, all respondents but two had established budgeted hourly rates for all presses. Both of the participants who did not have rates for their digital presses expressed interest in developing these rates in the near future.

When asked what percentage of their digital print work required a detailed cost-estimate, the responses varied significantly. Some companies produced estimates for nearly all their work while others rarely produced a cost-estimate. See Figure 2. 


\subsection{Estimating specific operations}

The next series of questions focused on the details of cost-estimating for commercial digital print, as follows:

- Describe the process you use to estimate the cost of database management.

- Describe the process you use to estimate the cost of digital asset management.

- Describe the process you use to estimate the cost of data cleansing, verification, and processing.

- Describe the process you use to estimate the cost of building a VDP template.

- Describe the process you use to estimate VDP RIPping.

- Do you use a different hourly rate for VDP output compared to short-run color digital printing?

Additional questions included:

- Do you offer response management services? If so, describe the process you use to estimate this service.

- Do you offer mailing services? If so, describe the process you use to estimate mailing.

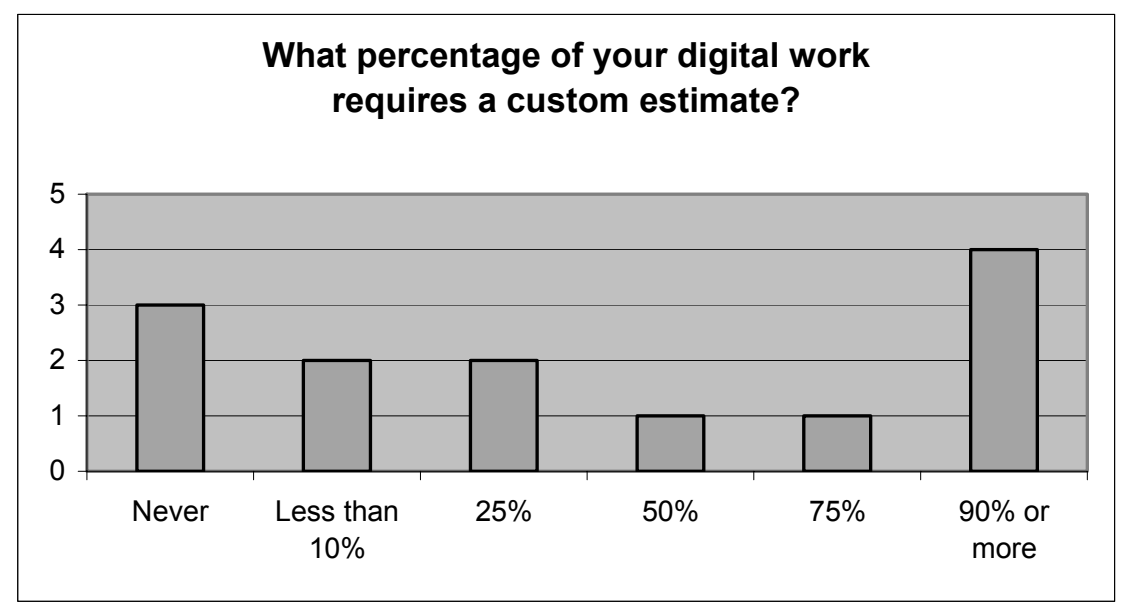

Fig. 2. Percentage of digital work requiring a custom estimate.

\subsubsection{Digital engines}

There are typically three equipment costs associated with operating digital print engines:

- Capital equipment expense (cost of the press/printer)

- Service agreement (monthly or yearly maintenance contract)

- Click charge (licensing agreement or royalty)

The click charge, popularized in the office copier market, involves a fee per impression paid to the press or printer manufacturer. The click charge may or may not include consumables and is normally discounted in tiers as volume increases. Sometimes, the lease contract includes a number of "clicks" per month, whether or not they are used.

Beyond these three equipment costs, other expenses may include labor, consumables, networking expenses, digital prepress equipment and VDP software, building modifications, training, and other expenses. 


\subsubsection{Digital front-end \& variable-data estimating}

Of all production functions, digital front-end workflow is one of the more difficult areas to estimate. For static digital print, prepress work is similar to commercial lithography. Variable-data printing, however, creates an additional set of challenges and costs for which the estimator must account. The discreet production steps that need estimating for VDP may include: renting lists; database and digital asset management; data cleansing, verification, and processing; building templates and data rules; RIPping; and response management. Some or all of these steps may be provided by the digital print manufacturer.

The digital printing company may be asked to secure data from a list broker. As such, the estimator must know multiple list suppliers and have the knowledge to intelligently discuss the needs of the client. Lists are typically rented on a per name or cost per thousand basis. The more refined or specialized the list, the more expensive it is.

If a clean list is provided, there may be little database management required. However, some customers may ask the printer to manage their database or to store digital assets used in marketing campaigns. Digital asset management (DAM) involves storing digital images for easy search and retrieval. The estimator should understand DAM production and must include the costs to build the DAM structure, manage the database, and include costs for search, retrieval, and use in collateral pieces.

Images used for VDP, whether stored as part of a DAM system or not, may require sizing, color management, cropping, or manipulation to ensure optimum results. Determining such needs in advance of a VDP job can be difficult unless sample files are provided. Clients must be advised of the specifications required for such images and notified that charges may be levied beyond the estimate if such manipulation is required.

Data cleansing, verification, and processing involves improving data quality and preparing it for use in VDP files. The most common data cleansing function is merge/purge. Merge/purge involves combining and eliminating duplicate entries. It is common for lists to contain two entries for some individuals, usually with slightly different name or address spellings. These should be fixed prior to production to avoid redundant mailings.

Data processing may also involve CASS certifying names for mailing. CASS (Coding Accuracy Support System) certification is a U.S. Postal Service requirement for receiving discounted postage. The certification process entails converting data entries into USPS approved format, and verification that each address falls within the known addresses for a particular street.

When asked about these functions, interviewees approached database work and digital asset management in varying ways. Only a handful of the research participants performed these functions in-house. When they did, they were handled on a price basis only - no structured cost information was computed. With complex data, estimators consult with their database expert to predict time needed to clean and prepare the database. Proofreading is necessary when $100 \%$ accuracy is required.

When asked about estimating for specific VDP prepress and data management operations, the responses varied significantly. Many companies handle these operations on a price list basis. Rather than estimate time or cost, a perpiece or flat charge is used.

Other respondents indicated that these operations were outsourced, simplifying the cost-estimating process. A few indicated that they use a "charge-back" system, where a price range is established and the customer is charged based on the actual complexity and time required to produce the work. One comment, "we quote static jobs, we estimate variable jobs."

Page building for static digital print is similar to the page layout process for conventional offset printing, while complex VDP work requires that dynamic fields be placed into the page. Templates and data rules (if/then statements) may be very complex and time-consuming to create, if not already in a library. From an estimating standpoint, page building for VDP may incorporate different tools and different hourly rates than static page layout. 
Estimating page template production and VDP rule building varied substantially depending on the complexity of the work produced. Nearly sixty percent estimated the time needed for these functions and multiplied by an hourly rate. One company simply charges a flat fee for VDP work, regardless of complexity. Three companies indicated that they do not charge additional fees for VDP prepress over static prepress, likely indicating they only produce low-complexity work. One company simply responded that the customer must provide the VDP files ready for output. See Figure 3.

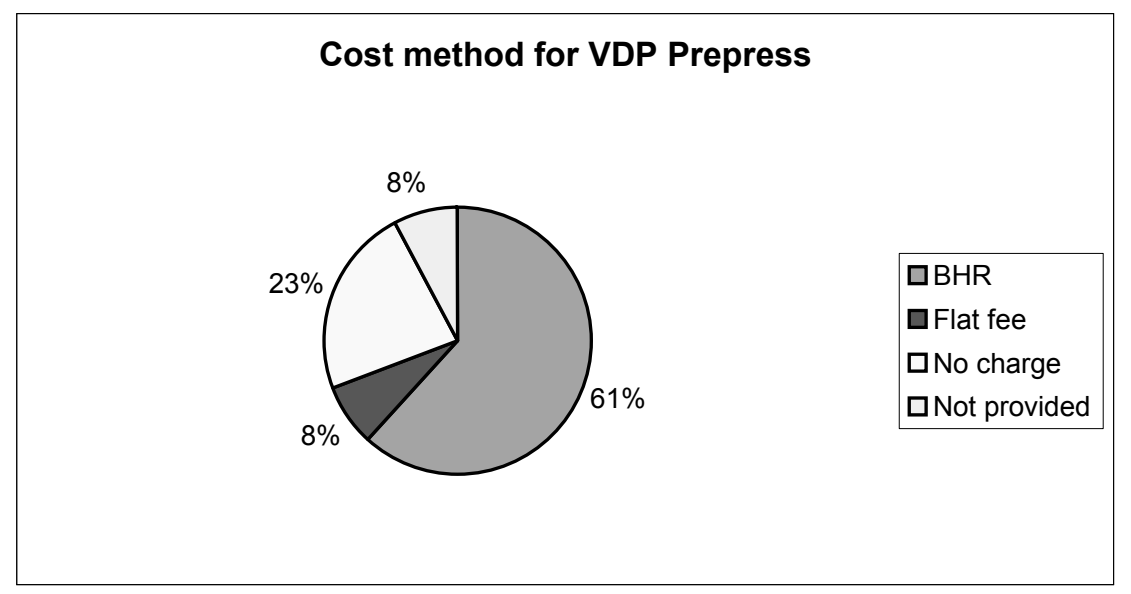

Fig. 3. Cost-estimating basis for variable-data printing prepress.

With VDP, processing data at the RIP (raster image processor) is an ongoing process, not a one-time event as in conventional printing. Complex or poorly-constructed VDP projects may take longer to process than is needed for the printing of each page, causing a delay in the delivery of output data to the print engine. This cost center may require multiple, specialized servers and advanced networking, thus increasing the hourly rate for VDP RIPping. Alternatives include a higher press hourly rate or simply applying a complexity factor for VDP work.

When asked if additional RIP costs are calculated for VDP work, there was little consistency in response. Just over half of the companies surveyed did not estimate additional RIP costs. One company estimated a flat percentage cost increase. Two companies estimated RIP time and multiplied by an hourly rate. Three companies charged a per piece RIP fee. See Figure 4. One company indicated that they will begin to incorporate a retainer or program fee for large VDP campaigns. This retainer fee is independent of print costs.

Price estimating is more common than cost-estimating for VDP prepress operations. For example, one company charged $\$ 0.02$ per side for VDP RIP processing. When asked if that reflects true costs and whether slow jobs are charged at a different rate than quick jobs, the answer was no. Typically, VDP prepress is price-listed.

\subsubsection{Mailing services and response management}

Significant consideration must be given to the careful handling of printed pieces in VDP for all downstream processes. Often, $100 \%$ completion is a contractual requirement, assuring that all individuals in a data list receive their document. Any pieces that are damaged, destroyed, or lost must be tracked, reprinted, and then finished before the project or program can be considered complete. The costs of such work must be estimated as a factor of waste within the project and included in the final price to the client. The more processes involved, the higher the potential waste may be. 
Most companies offer mailing services either in-house or by partnering with a mailing facility. When estimating for inhouse work, most companies use a staff mailing expert to estimate that portion of the job estimate. Postage is usually estimated as a separate line item.

The success of a VDP campaign is measured by response rates. Response management involves the tracking of responses or sales directly resulting from a VDP campaign. Marketers, retailers, and others who buy VDP must track and verify the success of one-to-one print campaigns. While this may be outside the purview of some companies, this value-added service may be provided by the digital printer in an effort to demonstrate return on investment (ROI) to the customer.

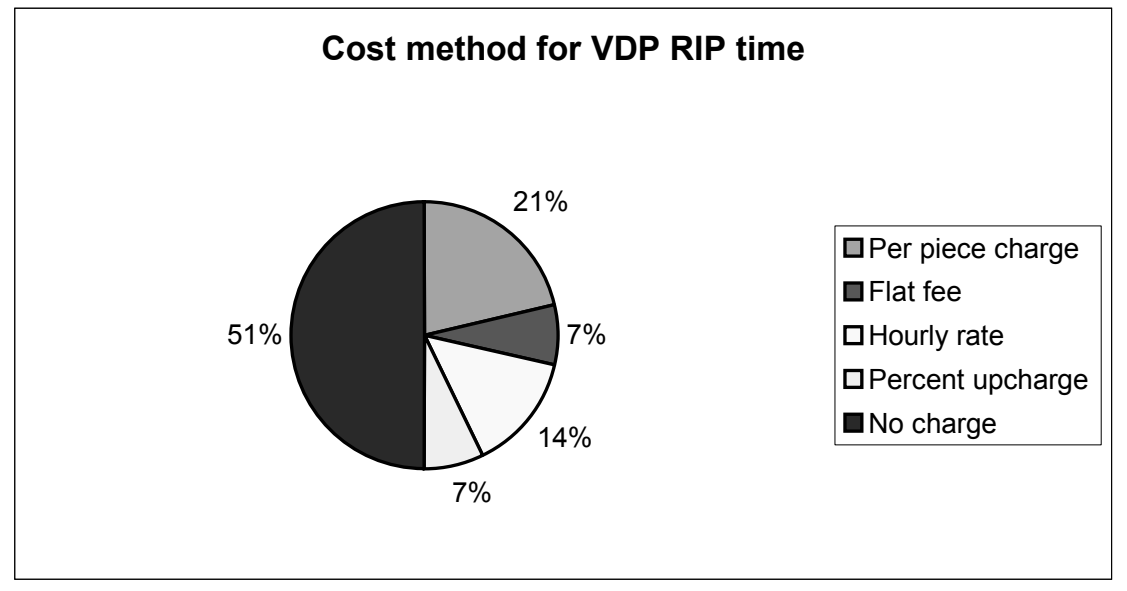

Fig. 4. Cost-estimating basis for variable-data printing RIP time.

When asked about response management, few participants are presently engaged in this activity. The minority, who perceive their role more as marketing firms offering consultative services, participate in response management, which includes developing ROI analyses and response tracking.

Two participants reported offering complete response management services, including processing response data, collecting money, and filling orders. One company provides this service to clients on a charge-back basis. The other company charges a set-up fee and a per-unit price for response management services.

\section{OBSERVATIONS AND CONCLUSIONS}

There were a number of observations derived from this research, including:

1. Digital printing companies experience better margins than conventional lithographers so there is less incentive to focus on cost calculations. Demonstrating response rates and return on investment is perceived as more important than focusing on costs.

2. As prices drop with more installations and the digital print market subsequently tightens, cost control and costestimating will increase in relevance. This is particularly true for low complexity work such as short-run color with minimal variation and finishing.

3. Database (list) management, cleansing, verification, and processing are highly price-list based. Few printers understand true costs associated with these functions.

4. The time required to build a page template with VDP rules can vary substantially by job and these costs can be difficult for the estimator to project. 


\subsection{Three approaches to digital printing}

There are three broad approaches to commercial digital printing:

- Traditional print providers, who supplement their offset presswork with digital printing for short-run color and versioned commercial print.

- "Low-touch" print providers, who leverage the power of the Internet to streamline business transactions with digital storefronts.

- Marketing solutions providers, who see printing less as a discrete manufacturing process and more as a component of a complete marketing campaign.

A company's approach will influence the need and relevance of an accurate estimating system. Traditional print providers and "low touch" providers need to have accurate job cost-estimating systems, as price models for these companies generally gravitate towards low-cost differentiation.

Traditional print providers already know how to cost-estimate, since most have been estimating offset work for some time. These companies may need to modify their estimating procedures to more accurately reflect digital printing characteristics. Conventional MIS estimating applications can adapt to short-run color engines. As more digital print engines enter a particular market, margins tighten and the work gets commoditized, increasing the need for cost control and estimating.

"Low touch" providers use a different approach. Their estimating needs involve immediate, computer-generated pricing through a digital storefront. Their pricing model prohibits customized estimating by a human. This work is lowcomplexity, highly-automated, and is well-suited for a pricing matrix.

The third category of digital printing companies, marketing solutions providers, focuses on consultative, complex services that have a greater pricing ceiling. While costs are important, the greater complexity, and subsequent highermargins, may reduce the relevance for a sound estimating process. When a company makes a lot of money, estimating, tracking, and controlling costs are perceived as unnecessary or secondary.

\subsection{Conclusions}

Traditional print providers use digital printing for short-run and versioned campaigns. These companies evolve from commercial lithographers, prepress providers, and photographers, and add digital engines to extend their capabilities into short-run lengths. They typically use an existing sales force and call on conventional printing clients. These companies use established estimating practices adapted from offset estimating.

Those with a conventional lithography background have a greater affinity to cost-estimating and tend to use costs as a basis for pricing. Those with a prepress or photography background lean toward price lists. Those with a marketing background understand the value of branding, cost-per-response, and look to perceived market value as a basis for pricing.

Pricing matrices are used liberally and may influence the commoditization of digital printing in the future, particularly in short-run color markets. The practice of offering substantial discounts on digital print jobs will continue to increase as competition grows.

"Low-touch" print providers leverage the power of the Internet to streamline business transactions with digital storefronts. These companies focus on very short-run and print-on-demand projects, reducing costs by limiting human contact with jobs. The dollar amount of each job is often quite low, necessitating a totally automated workflow. The onus is put on the customer to complete the job information, access online assets or upload a file for output, and initiate the order. 
The "low touch" model works well for short-run and very short-run static or versioned printing. Simple VDP work is possible too. "Low touch" companies use pricing matrices and real-time estimating via digital storefront to minimize the costs associated with producing an estimate for short-run digital printing. In many cases, the print transaction requires prepayment via credit card.

Driving costs out of administering a transaction is the key to success in static short-run digital printing. Reducing the "touch points" is critical. The primary way to drive costs out of the process is to automate the entire transaction through a Web portal, including pricing, job delivery, digital job ticket, preflighting, and job queuing/scheduling. For short-run color, job estimating and job costing are only cost-effective in situations when those processes can be automated. One participant suggests it costs $\$ 50$ to $\$ 100$ to complete a single estimate. One comment: "Can't stop and touch a job. If you stop to estimate it, you'll lose money."

Some digital press engines track and report costs, though there is some skepticism about how accurate these calculations are. For example, one participant complained that the tracking did not consider sales tax to be a cost and therefore inflated its profit calculations.

Marketing solutions providers see printing less as a discrete manufacturing process and more as a component of a comprehensive marketing campaign. These companies provide significant value by incorporating variable-data into widespread marketing programs. Web-to-print applications may be used, though the complexity of work is significantly higher than "low-touch" print providers. Marketing solutions companies focus on complex VDP projects and use project management teams to execute a marketing campaign. They function in a consultative role, focusing energy on tracking response and demonstrating ROI. VDP work requires greater quality control and more tracking of materials, generating higher costs throughout the process. These companies tend to use value-based pricing models focused on customer benefit rather than cost-estimating.

There is a big difference in the necessity for cost-based estimating in short-run color and print-on-demand where time and expenses are fairly predictable, compared to complex variable-data printing, where the intricacy of each job can substantially influence cost variation. Complex VDP work is difficult to estimate. A high-price is often negotiated for complex work and the printer makes money, though the precise amount of profit may not be known in advance.

\subsection{Final thoughts}

Understanding and predicting costs can be extremely beneficial for business decisions. Establishing a reliable system to estimate those costs can be somewhat challenging though. Estimating often takes a back seat, particularly when profit margins are strong. Unquestionably, cost-estimating digital printing will increase in relevance in the years ahead, as margins tighten and cost knowledge increases in value.

Before embarking on a cost-estimating system for digital printing, one must consider the ultimate goals. Is automating the estimating or pricing process important? Certainly if a company is striving to be "low touch," estimating must also be "low touch." If the objective is to produce precise estimates for cost containment, pricing matrices are not the best solution. Like production workflow, the estimating workflow for one company will not be the same as another. The first step is to determine the objective of such a system. With thought, a functional estimating process will help any company make better management, marketing, and production decisions. 